\title{
Differential-algebraic systems are generically controllable and stabilizable
}

\section{Achim Ilchmann $^{1}$ (D) Jonas Kirchhoff ${ }^{1}$}

Received: 16 November 2020 / Accepted: 27 April 2021 / Published online: 15 May 2021

(c) The Author(s) 2021

\begin{abstract}
We investigate genericity of various controllability and stabilizability concepts of linear, time-invariant differential-algebraic systems. Based on well-known algebraic characterizations of these concepts (see the survey article by Berger and Reis (in: Ilchmann A, Reis T (eds) Surveys in differential-algebraic equations I, DifferentialAlgebraic Equations Forum, Springer, Berlin, pp 1-61. https://doi.org/10.1007/9783-642-34928-7_1)), we use tools from algebraic geometry to characterize genericity of controllability and stabilizability in terms of matrix formats.
\end{abstract}

Keywords Differential-algebraic equations · Controllability · Stabilizability · Genericity

Mathematics Subject Classification 34A09 • 93B05

\section{List of symbols}

$\begin{array}{ll}\|x\|_{2} & :=\sqrt{x_{1}^{2}+\cdots+x_{n}^{2}}, \text { the Euclidean norm of } x=\left(x_{1}, \ldots, x_{n}\right) \in \mathbb{R}^{n} \\ \mathbb{N}, \mathbb{N}^{*} & :=\{0,1,2, \ldots\},:=\{1,2, \ldots\} \text { resp. } \\ \mathbb{R}, \mathbb{C} & \text { The field of the real, complex numbers, resp. } \\ \stackrel{\circ}{\mathbb{C}}_{-}, \overline{\mathbb{C}}_{+} & :=\{z \in \mathbb{C} \mid \Re z<0\}, \text { the open left (resp. closed right) half plane } \\ & :=\{1, \ldots, j\} \geq 0\} ? \\ \underline{j}^{-1}(A) & :=\{x \in X: f(x) \in A\}, \text { the preimage of the set } A \subseteq Y \text { under the } \\ & \text { function } f: X \rightarrow Y . \\ \lambda^{n} & \text { The Lebesgue measure on } \mathbb{R}^{n} .\end{array}$

Achim Ilchmann

achim.ilchmann@tu-ilmenau.de

Jonas Kirchhoff

jonas.kirchhoff@tu-ilmenau.de

1 Institut für Mathematik, Technische Universität Ilmenau, Weimarer Straße 25, 98693 Ilmenau, Germany 
$R^{k \times \ell}$

$\mathrm{rk}_{F} M$

$\left[\mathbb{R} x_{1}, \ldots, x_{n}\right]$

$\mathbb{R}\left(x_{1}, \ldots, x_{n}\right)$

The vector space of all $k \times \ell$ matrices with entries in a ring $R$. The rank of $M \in R^{k \times \ell}$ over the field $F$.

$:=\left\{\sum_{k=0}^{\ell} a_{k} x_{1}^{\nu_{k, 1}} \cdots x_{n}^{\nu_{k, n}} \mid \ell \in \mathbb{N}, a_{k} \in \mathbb{R}, v_{k, j} \in \mathbb{N}\right\}$, the ring of (real) polynomials in $n$ indeterminants.

\section{Introduction}

We study genericity of controllability and stabilizability of differential algebraic systems described by the equation

$$
\frac{\mathrm{d}}{\mathrm{d} t}(E x)=A x+B u,
$$

where

$$
(E, A, B) \in \quad \Sigma_{\ell, n, m}:=\mathbb{R}^{\ell \times n} \times \mathbb{R}^{\ell \times n} \times \mathbb{R}^{\ell \times m} .
$$

To be precise, we first say what we understand under genericity.

Definition 1.1 [13, p. 28] and [11, p. 50] A set $\mathbb{V} \subseteq \mathbb{R}^{n}$ is called an algebraic variety, if there exist finitely many polynomials

$$
p_{1}\left(x_{1}, \ldots, x_{n}\right), \ldots, p_{k}\left(x_{1}, \ldots, x_{n}\right) \in \mathbb{R}\left[x_{1}, \ldots, x_{n}\right]
$$

such that $\mathbb{V}$ is the locus of their zeros, i.e.,

$$
\mathbb{V}=\left\{x \in \mathbb{R}^{n} \mid \forall i \in \underline{k}: p_{i}(x)=0\right\}=\bigcap_{i=1}^{k} p_{i}^{-1}(\{0\})
$$

An algebraic variety $\mathbb{V}$ is called proper if $\mathbb{V} \subsetneq \mathbb{R}^{n}$. The set of all algebraic varieties in $\mathbb{R}^{n}$ is denoted as

$$
\mathscr{V}_{n}(\mathbb{R}):=\left\{\mathbb{V} \subseteq \mathbb{R}^{n} \mid \exists q_{1}(\cdot), \ldots, q_{k}(\cdot) \in \mathbb{R}\left[x_{1}, \ldots, x_{n}\right]: \bigcap_{i=1}^{k} q_{i}^{-1}(\{0\})=\mathbb{V}\right\}
$$

and the set of all proper algebraic varieties as

$$
\mathscr{V}_{n}^{\text {prop }}(\mathbb{R}):=\mathscr{V}_{n}(\mathbb{R}) \backslash\left\{\mathbb{R}^{n}\right\}
$$

A set $S \subseteq \mathbb{R}^{n}$ is called generic, if there exists a proper algebraic variety $\mathbb{V} \in \mathscr{V}_{n}^{\text {prop }}(\mathbb{R})$ so that $S^{c} \subseteq \mathbb{V}$. If the algebraic variety $\mathbb{V}$ is known, then we call $S$ generic with respect to (w.r.t.) $\mathbb{V}$. 
"Generic" is not consistently used in the literature. We show in the following proposition that generic as in Definition 1.1 is stronger than containing an open and dense subset with respect to the Euclidean topology.

Proposition 1.2 Any set $S \subseteq \mathbb{R}^{n}$ satisfies:

(i) If $\mathbb{V} \subseteq \mathbb{R}^{n}$ is a proper algebraic variety, then $\mathbb{V}^{c}$ is open and dense.

(ii) If $S \subseteq \mathbb{R}^{n}$ is generic w.r.t. $\mathbb{V} \in \mathscr{V}_{n}^{\text {prop }}(\mathbb{R})$, then $S$ contains the open and dense subset $\mathbb{V}^{c}$.

(iii) There exists an open and dense set $S \subseteq \mathbb{R}^{n}$ with $\lambda^{n}(S)<\infty$, and therefore $S$ is neither generic nor is $S^{c}$ a proper algebraic variety. In other words, the reverse implications in (i) and (ii) do not hold true.

Proof (i) We show that $\mathbb{V}$ is closed. Let $\mathbb{V}$ be given as in (2). Then, each $p_{i}^{-1}(\{0\})$ is closed since $p_{i}$ is continuous and hence, the claim follows.

It remains to prove that $\mathbb{V}^{c}$ is dense. Seeking a contradiction, suppose that $\mathbb{V}^{c}$ is not dense or, equivalently, $\mathbb{V}$ has at least one inner point. Then, $\lambda^{n}(\mathbb{V})>0$ and $\mathbb{V}$ cannot be a proper algebraic variety by Proposition A.3.

(ii) This is an immediate consequence of (i).

(iii) Let $\varphi: \mathbb{N} \rightarrow \mathbb{Q}^{n}$ be a bijection and set

$$
S:=\bigcup_{i \in \mathbb{N}}\left\{q \in \mathbb{R}^{n} \mid\|q-\varphi(i)\|<42^{-i}\right\} \supseteq \mathbb{Q}^{n} .
$$

Then, $S \subseteq \mathbb{R}^{n}$ is open, dense and satisfies

$$
\lambda^{n}(S) \leq \sum_{i \in \mathbb{N}} \frac{2^{n}}{42^{i n}} \leq 2^{n} \sum_{i \in \mathbb{N}} \frac{1}{42^{i}}=2^{n} \frac{42}{41}<\infty .
$$

Now, Lemma A.7 shows that $S$ is not generic.

If $S^{c}=\mathbb{V}$ were an algebraic variety, then $S$ is generic w.r.t. $\mathbb{V}$ which is a contradiction. This completes the proof.

To characterize genericity in terms of the Zariski topology, recall [11, p.50] that the latter is defined by the property that all closed sets are the algebraic varieties. The Zariski topology is strictly coarser than the Euclidean topology and we have: A set $S \subseteq \mathbb{R}^{n}$ is generic if, and only if, $S$ contains a nonempty Zariski open set.

This approach was used by Belur and Shankar in their investigations of genericity of impulse controllable systems (see [3, Section 3]). Since they consider differentialalgebraic equations described by differential operator matrices and hence an infinite dimensional vector space, they need to extend the definition of generic sets to this space using the limit topology of the Zariski topology. This is not necessary in our setup.

In the special case that (1) is an ordinary differential equation, that is $\ell=n$ and $E=I$, Lee and Markus [10] proved that that the set of all controllable systems is open and dense w.r.t. the Euclidean topology. Wonham [12, Thm. 1.3] showed in the first edition of his monograph that the set of all controllable systems is generic. 
Recently, it has been shown that linear, time-invariant port-Hamiltonian systems are generically controllable; see [9].

When it comes to differential-algebraic equations, then to the best of our knowledge there are only two contributions known where open and dense subsets of controllable systems are investigated. Banaszuk and Przyłuski [1] consider an algebraic criterionwhich they do not justify analytically and which is not related to any concept of controllability - and give a sufficient condition so that the set of systems satisfying the algebraic criterion contains an open and dense subset. The second contribution is by Belur and Shankar [3].

Their main interest is on polynomial systems, and if specialized to matrix pencils, they derive a characterization of genericity of impulse controllability. Other concepts are not studied.

The basis of our approach is the algebraic characterizations of various concepts of controllability and stabilizability of differential-algebraic equations; this is well known and summarized in Propositions 2.1 and 3.1. We characterize-in terms of the formats $\ell, n$, and $m$ of (1)—when these controllability and stabilizability concept hold generically. This is the content of Theorems 2.3 and 3.3. The proofs of these two main results are based on methods from algebraic geometry, tailored for our purposes and relegated to "Appendix 1", and some results on ranks of special matrices are presented in "Appendix 2".

\section{Controllability}

There are various controllability concepts for differential-algebraic equations (1) such as freely initializable (also called 'controllable at infinity'), impulse controllable, completely controllable, behavioral controllable, and strongly controllable. Their definitions and their algebraic characterizations are given in the next proposition. To state this, we need to say what a solution of (1) is. We consider the behavior of (1) given by

$$
\mathfrak{B}_{[E, A, B]}:=\left\{\begin{array}{l|l}
(x, u) \in \mathscr{W}_{\mathrm{loc}}^{1,1}\left(\mathbb{R}, \mathbb{R}^{n}\right) \times \mathscr{L}_{\mathrm{loc}}^{1}\left(\mathbb{R}, \mathbb{R}^{m}\right) & \begin{array}{l}
E x \text { is absolutely continuous } \\
\text { and for a.a. } t \in \mathbb{R}: \\
\frac{\mathrm{d}}{\mathrm{d} t}(E x)(t)=A x(t)+B u(t)
\end{array}
\end{array}\right\}
$$

where $\mathscr{L}_{\text {loc }}^{1}\left(\mathbb{R}, \mathbb{R}^{d}\right)$ denotes the set of locally integrable functions $f: \mathbb{R} \rightarrow \mathbb{R}^{d}$, and $\mathscr{W}_{\mathrm{loc}}^{1,1}\left(\mathbb{R}, \mathbb{R}^{d}\right)$ is the Sobolev space of all functions $f \in \mathscr{L}_{\mathrm{loc}}^{1}\left(\mathbb{R}, \mathbb{R}^{d}\right)$ with $f^{(1)} \in$ $\mathscr{L}_{\text {loc }}^{1}\left(\mathbb{R}, \mathbb{R}^{d}\right)$. Note that any $f \in \mathscr{W}_{\mathrm{loc}}^{1,1}\left(\mathbb{R}, \mathbb{R}^{d}\right)$ is continuous.

Controllability of a system (1) is a property of the corresponding behavior $\mathfrak{B}_{[E, A, B]}$. If systems described by ordinary differential equations are considered, i.e., the special case $E=I$, then the initial value $x^{0} \in \mathbb{R}^{n}$ can be freely chosen and the problem is to which other points it can be steered in finite time. It is well-known that the system (1) with $E=I$ is called controllable if, and only if, for any given initial state $x^{0} \in$ $\mathbb{R}^{n}$ and any terminal state $x^{1} \in \mathbb{R}^{n}$, there exists a control $u \in \mathscr{L}_{\text {loc }}^{1}\left(\mathbb{R}, \mathbb{R}^{m}\right)$ which 
steers $x^{0}$ to $x^{1}$ in finite time $T>0$, more formally, there exists $(x, u) \in \mathfrak{B}_{[E, A, B]}$ such that $x(0)=x^{0}$ and $x(T)=x^{1}$.

However, if an arbitrary matrix $E$ is allowed in (1), then algebraic constraints are added to the differential equation. So it is unclear as to whether the initial value can be chosen freely. If the latter is the case, then the system is called freely initializable, sometimes also called controllable at infinity.

If (1) is assumed to be freely initializable, then one may ask, whether each initial state can be steered to any final state in finite time. If both conditions are fulfilled, then the system is called completely controllable.

A stronger controllability concept — but also a generalization of the ODE case-is in the behavioral setup the problem as to whether it is always possible to concatenate two given solutions $\left(x_{1}, u_{1}\right),\left(x_{2}, u_{2}\right) \in \mathfrak{B}_{[E, A, B]}$ by another solution $(x, u) \in \mathfrak{B}_{[E, A, B]}$ over the time interval $[0, T]$. Such systems are called behavioral controllable systems.

The concepts of freely initializable and completely controllable systems can be weakened in the sense that the initial and the terminal value are compared with respect to the image of $E$; for example, the initial condition becomes $E x^{0}=E x(0)$. These weakened concepts are called impulse controllable and strongly controllable.

The precise definitions and algebraic characterizations are given in the following proposition.

Proposition 2.1 For any $(E, A, B) \in \Sigma_{\ell, n, m}$, the following controllability definitions associated with the system (1) are algebraic characterized as follows:

$$
\begin{aligned}
& \text { freely initializable } \quad:=\forall x^{0} \in \mathbb{R}^{n} \exists(x, u) \in \mathfrak{B}_{[E, A, B]}: x(0)=x^{0} \\
& \Longleftrightarrow \mathrm{rk}[E, B]=\mathrm{rk}[E, A, B] \text {; } \\
& \text { impulse controllable } \quad:=\forall x^{0} \in \mathbb{R}^{n} \exists(x, u) \in \mathfrak{B}_{[E, A, B]}: E x^{0}=E x(0) \\
& \Longleftrightarrow \forall Z \in \mathbb{R}^{n \times n-\mathrm{rk} E} \text { with } \operatorname{im}_{\mathbb{R}} Z=\operatorname{ker}_{\mathbb{R}} E \\
& \text { : } \operatorname{rk}[E, A, B]=\operatorname{rk}[E, A Z, B] \text {; } \\
& \text { behavioral controllable }:=\quad \forall\left(x_{1}, u_{1}\right),\left(x_{2}, u_{2}\right) \in \mathfrak{B}_{[E, A, B]} \exists T>0 \exists(x, u) \in \mathfrak{B}_{[E, A, B]} \\
& :(x, u)(t)= \begin{cases}\left(x_{1}, u_{1}\right)(t), & t<0 \\
\left(x_{2}, u_{2}\right)(t), & t>T\end{cases} \\
& \Longleftrightarrow \forall \lambda \in \mathbb{C}: \operatorname{rk}_{\mathbb{R}(s)}[s E-A, B]=\operatorname{rk}_{\mathbb{C}}[\lambda E-A, B] ; \\
& \text { completely controllable }:=\quad \exists T>0 \forall x^{0}, x_{T} \in \mathbb{R}^{n} \exists(x, u) \in \mathfrak{B}_{[E, A, B]} \\
& : x(0)=x^{0} \wedge x(T)=x_{T} \\
& \Longleftrightarrow \forall \lambda \in \mathbb{C}: \operatorname{rk}[E, A, B]=\operatorname{rk}[E, B]=\operatorname{rk}[\lambda E-A, B] ; \\
& \text { strongly controllable }:=\quad \exists T>0 \forall x^{0}, x_{T} \in \mathbb{R}^{n} \exists(x, u) \in \mathfrak{B}_{[E, A, B]} \\
& : \operatorname{Ex}(0)=E x^{0} \wedge \operatorname{Ex}(T)=E x_{T} \\
& \Longleftrightarrow \forall \lambda \in \mathbb{C} \forall Z \in \mathbb{R}^{n \times n-\mathrm{rk} E} \text { with im } \mathbb{R}_{\mathbb{R}} Z=\operatorname{ker}_{\mathbb{R}} E \\
& : \operatorname{rk}[E, A, B]=\operatorname{rk}[E, A Z, B]=\operatorname{rk}[\lambda E-A, B] \text {. }
\end{aligned}
$$

Proof Berger and Reis [4] derive a feedback form and use this as a tool in conjunction with 'canonical' representatives of certain equivalence classes to prove all characterizations of controllability in their survey. Note that in their characterization of strongly controllability, the term ' $+\mathrm{im}_{\mathbb{R}} E$ ' is missing in the first respective line in [4, Cor. 4.3]. 
Remark 2.2 The equivalences

$$
(E, A, B) \text { str. contr. } \Longleftrightarrow(E, A, B) \text { imp. contr. and beh. contr. }
$$

and

$(E, A, B)$ compl. contr. $\Longleftrightarrow(E, A, B)$ freely initial. and beh. contr.

are proved in [4, Rem. 4.4].

Genericity of the different controllability concepts can be characterized in terms of the system dimensions. To this end, we introduce the notation

$$
S_{\text {controllable }}:=\left\{(E, A, B) \in \Sigma_{\ell, n, m} \mid(1) \text { is controllable }\right\}
$$

where 'controllable' stands for one of the controllability concepts.

Theorem 2.3 For each of the controllability concepts defined in Proposition 2.1, the following characterizations hold:

$\begin{array}{llll}S_{\text {freely initial. }} \text { is generic } & \Longleftrightarrow & \ell \leq n+m ; \\ S_{\text {imp. contr. }} \text { is generic } & \Longleftrightarrow & \ell \leq n+m ; \\ S_{\text {beh. contr. }} \text { is generic } & \Longleftrightarrow & \ell \neq n+m ; \\ S_{\text {compl. contr. } \text { is generic }} \Longleftrightarrow & \Longleftrightarrow \ell<n+m ; \\ S_{\text {strongly. contr. is generic }} \Longleftrightarrow \ell & \ell<n+m .\end{array}$

Proof We proceed in steps.

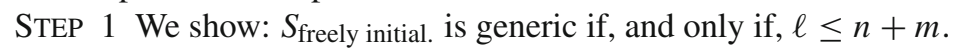

First note that Proposition 2.1 yields

$S_{\text {freely initial. }}=\left\{(E, A, B) \in \Sigma_{\ell, n, m} \mid \operatorname{rk}[E, A, B]=\operatorname{rk}[E, B]\right\}$.

$\Longrightarrow$ Let $\ell>n+m$. By Proposition B.3 (i) and (ii), the sets

$$
\begin{aligned}
S_{(\mathrm{ii})} & :=\left\{(E, A, B) \in \Sigma_{\ell, n, m} \mid \operatorname{rk}[E, B]=n+m\right\}, \\
S_{(\mathrm{i})} & :=\left\{(E, A, B) \in \Sigma_{\ell, n, m} \mid \operatorname{rk}[E, A, B]=\min \{\ell, 2 n+m\}\right\} .
\end{aligned}
$$

are generic sets. Hence, Corollary A.5(ii) implies that $S_{(\mathrm{i})} \cap S_{(\mathrm{ii})}$ is a generic set. If $(E, A, B) \in S_{(\mathrm{i})} \cap S_{(\mathrm{ii})}$, then $\ell>n+m$ yields

$$
\operatorname{rk}[E, B]=n+m<\min \{\ell, 2 n+m\}=\operatorname{rk}[E, A, B]
$$

and therefore $S_{(\mathrm{i})} \cap S_{\text {(ii) }} \subseteq S_{\text {freely initial. }}^{c}$ and $S_{\text {freely initial. }}^{c}$ is generic. Thus, Lemma A.6 shows that $S_{\text {freely initial. }}$ is not generic.

$\Longleftarrow$ Since $\ell \leq n+m$, the sets

$$
\begin{aligned}
S_{(\mathrm{ii})} & :=\left\{(E, A, B) \in \Sigma_{\ell, n, m} \mid \operatorname{rk}[E, B]=\ell\right\}, \\
S_{(\mathrm{i})} & :=\left\{(E, A, B) \in \Sigma_{\ell, n, m} \mid \operatorname{rk}[E, A, B]=\ell\right\}
\end{aligned}
$$


are both non-empty and by Proposition B.3 (i) and (ii) they are generic. Now, Corollary A.5(ii) yields that $S_{(\mathrm{i})} \cap S_{(\mathrm{ii})}$ is a generic set, and by Remark A.1, $S_{\text {freely initial. }} \supseteq$ $S_{1} \cap S_{2}$ is generic, too.

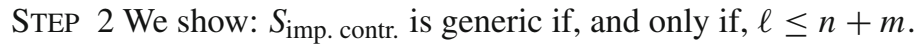

We consider the two cases $\ell \geq n$ and $\ell<n$.

$\ell \geq n$ : By Proposition B.3 (i) and (iii), the sets

$$
\begin{array}{rl|l}
S_{(\mathrm{i})} & :=\left\{(E, A, B) \in \Sigma_{\ell, n, m}\right. & \operatorname{rk}[E, A, B]=\min \{\ell, 2 n+m\}\}, \\
S_{(\mathrm{iii})} & :=\left\{(E, A, B) \in \Sigma_{\ell, n, m}\right. & \operatorname{rk} E=n\}
\end{array}
$$

are generic and thus Corollary A.5 implies that $S_{(\mathrm{i})} \cap S_{(\mathrm{iii})}$ is also generic. For each $(E, A, B) \in S_{2}$, we find by the rank-nullity theorem that ker $E=\{0\}$. Hence, Proposition 2.1 yields that

$$
S_{\text {imp. contr. }} \cap S_{\text {(i) }} \cap S_{\text {(iii) }}=\underbrace{\left\{(E, A, B) \in \Sigma_{n, n, m} \mid \operatorname{rk}[E, B]=\min \{\ell, 2 n+m\}\right\}}_{=: \widetilde{S}} \cap S_{(\mathrm{i})} \cap S_{(\mathrm{iii})} .
$$

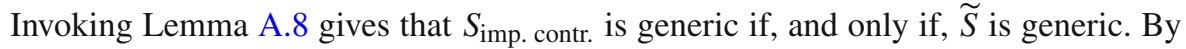
Proposition B.3, this is the case if, and only if, $\min \{\ell, 2 n+m\} \leq \min \{\ell, n+m\}$. This inequality holds true if, and only if, $\ell \leq n+m$.

$\ell<n: \quad$ Put $\widehat{S}:=\left\{(E, A, B) \in \Sigma_{n, n, m} \mid \operatorname{rk}[E, B]=\ell=\min \{\ell, n+m\}\right\}$, which is generic by Proposition B.3 (ii). Note that both statements

$$
\begin{aligned}
& \forall(E, A, B) \in \Sigma_{\ell, n, m} \forall Z \in \mathbb{R}^{n \times n-\mathrm{rk} E} \text { with } \operatorname{im}_{\mathbb{R}} Z=\operatorname{ker}_{\mathbb{R}} E: \operatorname{rk}[E, A Z, B] \leq \ell, \\
& \quad \forall(E, A, B) \in \widehat{S} \quad \forall Z \in \mathbb{R}^{n \times n-\mathrm{rk} E} \text { with } \operatorname{im}_{\mathbb{R}} Z=\operatorname{ker}_{\mathbb{R}} E: \operatorname{rk}[E, A Z, B]=\ell
\end{aligned}
$$

hold true. By Proposition 2.1, we find that $\widehat{S} \cap S_{(i)]} \subseteq S_{\text {imp. contr. and hence, in view }}$ of Corollary A.5(ii) and Remark A.1, the set $S_{\text {imp. contr. }}$ is generic.

STEP 3 Proposition 2.1 yields

$$
S_{\text {beh. contr. }}=\left\{\begin{array}{l|l}
(E, A, B) \in \Sigma_{\ell, n, m} & \begin{array}{l}
\forall \lambda \in \mathbb{C}: \operatorname{rk}_{\mathbb{R}(s)}[s E-A, B] \\
=\operatorname{rk}_{\mathbb{C}}[\lambda E-A, B]
\end{array}
\end{array}\right\} .
$$

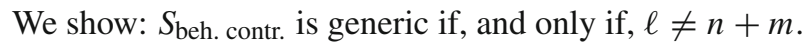

From Proposition B.5, we find that the set

$$
S_{3}:=\left\{(E, A, B) \in \Sigma_{\ell, n, m} \mid \operatorname{rk}_{\mathbb{R}(s)}[s E-A, B]=\min \{\ell, n+m\}\right\}
$$

is a generic set. The equation (5) implies

$S_{\text {beh. contr. }} \cap S_{3}=\left\{(E, A, B) \in \Sigma_{\ell, n, m} \mid \forall \lambda \in \mathbb{C}: \operatorname{rk} \mathbb{C}[\lambda E-A, B]=\min \{\ell, n+m\}\right\}$.

By Lemma A.8, genericity of $S_{\text {beh. contr. }} \cap S_{3}$ is necessary and sufficient for $S_{\text {beh. contr. }}$ being generic. Now, Proposition B.8 gives: $S_{\text {beh. contr. }} \cap S_{1}$ is generic if, and only if, $\ell \neq n+m$. 
STEP 4 Since

$$
S_{\text {strongly contr. }}=S_{\text {imp. contr. }} \cap S_{\text {beh. contr. }}
$$

Corollary A.5 (ii) implies that $S_{\text {strongly contr. }}$ is generic if, and only if, both $S_{\text {imp. contr. }}$

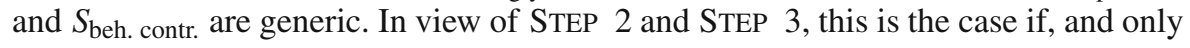
if, $\ell<n+m$.

STEP 5 Applying Corollary A.5 (ii) to the equality

$$
S_{\text {compl. contr. }}=S_{\text {freely initial. }} \cap S_{\text {beh. contr. }}
$$

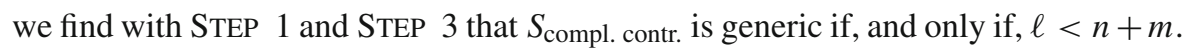

In the following remark, we stress the observation that linear differential-algebraic systems are either generically controllable or generically not controllable.

Remark 2.4 A closer inspection of the proof of Theorem 2.3 yields that if $S_{\text {arbitrary controllability }}$ is not generic, then it is contained in a proper algebraic variety and thus its complement is generic.

\section{Stabilizability}

In the present section, genericity of stabilizability of DAEs is studied.

In the ODE-case, a system (1) is called stabilizable if, and only if, for each initial value $x^{0} \in \mathbb{R}^{n}$ there exists a control $u \in \mathscr{L}_{\text {loc }}^{1}\left(\mathbb{R}, \mathbb{R}^{m}\right)$ which steers the forced trajectory $x$ in (possibly) infinite time to zero, that is $(x, u) \in \mathfrak{B}_{[I, A, B]}$ so that $x(0)=x^{0}$ and $\lim _{t \rightarrow \infty}$ ess sup $x|(t, \infty)|=0$.

As for controllability, different generalizing concepts have to be studied for DAEs. Each system that is stabilizable in the ODE-sense is called completely stabilizable. Similar to controllability, this concept is weakened if only $E x(t)$ is considered-in this case we speak of strong stabilizability.

Finally, a system is called behavioral controllable if, and only if, each $(x, u) \in$ $\mathfrak{B}_{[E, A, B]}$ can be concatenated with some $(x, u) \in \mathfrak{B}_{[E, A, B]}$ which tends to zero as $t$ tends to infinity.

The precise definitions and algebraic characterizations are given in the following proposition. We write $\mathscr{W}_{\text {loc }}^{1,1}\left(I, \mathbb{R}^{n}\right)$ for the set of all weakly differentiable $\varphi \in \mathscr{L}_{\mathrm{loc}}^{1}\left(\mathbb{R}, \mathbb{R}^{n}\right)$. 
Proposition 3.1 For any $(E, A, B) \in \Sigma_{\ell, n, m}$, the following controllability definitions associated with the system (1) are algebraic characterized as follows:

$$
\begin{aligned}
\text { compl. stabl. }:= & \forall x^{0} \in \mathbb{R}^{n} \exists(x, u) \in \mathfrak{B}_{[E, A, B]} x(0)=x^{0} \wedge \lim _{t \rightarrow \infty} \text { ess sup } x_{(t, \infty)}=0 \\
\Longleftrightarrow & \forall \lambda \in \overline{\mathbb{C}}_{+}: \operatorname{rk}[E, A, B]=\operatorname{rk}[E, B]=\operatorname{rk}[\lambda E-A, B] ; \\
\text { str. stabl. }:= & \forall x^{0} \in \mathbb{R}^{n} \exists(x, u) \in \mathfrak{B}_{[E, A, B]}: E x(0)=E x^{0} \wedge \lim _{t \rightarrow \infty} E x(t)=0 \\
\Longleftrightarrow & \forall \lambda \in \overline{\mathbb{C}}_{+} \forall Z \text { with } \operatorname{im} Z=\operatorname{ker} E \\
& : \operatorname{rk}[E, A, B]=\operatorname{rk}[E, A Z, B]=\operatorname{rk}[\lambda E-A, B] ; \\
\text { beh. stabl. }:= & \forall(x, u) \in \mathfrak{B}_{[E, A, B]} \exists\left(x_{1}, u_{1}\right) \in \mathfrak{B}_{[E, A, B]} \cap\left(\mathscr{W}_{\operatorname{loc}^{1,1}}\left(\mathbb{R}, \mathbb{R}^{n}\right) \times \mathscr{W}_{\operatorname{loc}}^{1,1}\left(\mathbb{R}_{,} \mathbb{R}^{m}\right)\right) \\
& :\left[\forall t<0:(x(t), u(t))=\left(x_{1}(t), u_{1}(t)\right)\right] \wedge \lim _{t \rightarrow \infty}\left(x_{1}(t), u_{1}(t)\right)=0 \\
\Longleftrightarrow & \forall \lambda \in \overline{\mathbb{C}}_{+}: \operatorname{rk} \mathbb{R}(s)[s E-A, B]=\operatorname{rk} \mathbb{C}[\lambda E-A, B] .
\end{aligned}
$$

Proof All characterizations are proved in the survey article by Berger and Reis [4, Cor. 4.3].

Remark 3.2 The equivalences

$$
(E, A, B) \text { compl. stabl. } \Longleftrightarrow(E, A, B) \text { beh. stab. and freely init. }
$$

and

$$
(E, A, B) \text { str. stabl. } \Longleftrightarrow(E, A, B) \text { beh. stab. and str. contr. }
$$

are proved in [4, Rem. 4.5].

We now show how genericity of the different stability concepts can be characterized in terms of the matrix dimensions. To this end, we introduce the notion

$$
S_{\text {stabliizable }}:=\left\{(E, A, B) \in \Sigma_{\ell, n, m} \mid \text { (1) stabilizable }\right\}
$$

where 'stabilizable' stands for one of the stability concepts.

Theorem 3.3 For each of the three stabilizability concepts from Proposition 3.1, the following characterizations hold:

$$
\begin{array}{llll}
S_{\text {beh. stab. }} \text { is generic } & \Longleftrightarrow & \ell \neq n+m ; \\
S_{\text {compl. stab. }} \text { is generic } & \Longleftrightarrow \quad \ell<n+m ; \\
S_{\text {strongly stab. } \text { is generic }} \Longleftrightarrow \ell \ell<n+m .
\end{array}
$$

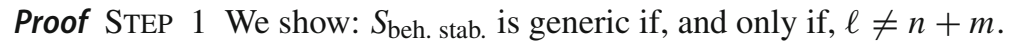

Proposition B.5 yields that the set

$$
S_{1}:=\left\{(E, A, B) \in \Sigma_{\ell, n, m} \mid \operatorname{rk}_{\mathbb{R}(s)}[s E-A, B]=\min \{\ell, n+m\}\right\}
$$

is generic. By Lemma A.8, genericity of

$$
S_{2}:=\left\{(E, A, B) \in \Sigma_{\ell, n, m} \mid \forall \lambda \in \overline{\mathbb{C}}_{+}: \operatorname{rk} \mathbb{C}[\lambda E-A, B]=\min \{\ell, n+m\}\right\}
$$


is a necessary and sufficient condition for genericity of $S_{\text {beh. stab. }}$ By Proposition B.9, $S_{2}$ is generic if, and only if, $\ell \neq n+m$.

STEP 2 Remark 3.2 yields that

$$
S_{\text {compl. stabl. }}=S_{\text {beh. stabl. }} \cap S_{\text {freely init. }}
$$

Thus, Corollary A.5 (ii) together with Theorem 2.3 gives that $S_{\text {compl. stabl. is generic }}$ if, and only if, $\ell<n+m$.

STEP 3 Applying Corollary A.5 (ii) and Theorem 2.3 to the equality

$$
S_{\text {str. stabl. }}=S_{\text {beh. stabl. }} \cap S_{\text {str. contr. }}
$$

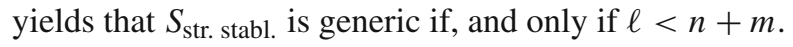

As for controllability, we would like to emphasize that linear differential-algebraic systems are either generically controllable or generically not controllable.

Remark 3.4 A closer inspection of the proof of Theorem 3.3 yields as in Remark 2.4 that $S_{\text {stabilizable }}$ is either generic or contained in a proper algebraic variety.

Acknowledgements We are indebted to our colleague Thomas Hotz (Ilmenau) for several constructive discussions, and to a reviewer who read the two submissions very carefully and made many helpful comments.

Funding Open Access funding enabled and organized by Projekt DEAL.

Open Access This article is licensed under a Creative Commons Attribution 4.0 International License, which permits use, sharing, adaptation, distribution and reproduction in any medium or format, as long as you give appropriate credit to the original author(s) and the source, provide a link to the Creative Commons licence, and indicate if changes were made. The images or other third party material in this article are included in the article's Creative Commons licence, unless indicated otherwise in a credit line to the material. If material is not included in the article's Creative Commons licence and your intended use is not permitted by statutory regulation or exceeds the permitted use, you will need to obtain permission directly from the copyright holder. To view a copy of this licence, visit http://creativecommons.org/licenses/by/4.0/.

\section{Appendix A Algebraic geometry}

The results presented in the present section are tailored from algebraic geometry for our needs. They provide a basis for the proofs of Theorems 2.3 and 3.3.

Throughout this section, let $n \in \mathbb{N}^{*}$.

We identify, wherever needed, any polynomial—an algebraic object-

$$
p(x)=p\left(x_{1}, \ldots, x_{n}\right)=\sum_{k=0}^{\ell} a_{k} x_{1}^{v_{k, 1}} \cdots x_{n}^{v_{k, n}} \in \mathbb{R}\left[x_{1}, \ldots, x_{n}\right]
$$

with the polynomial - an algebraic object -

$$
p(\cdot): \mathbb{R}^{n} \rightarrow \mathbb{R}, \quad x=\left(x_{1}, \ldots, x_{n}\right) \mapsto p(x)=\sum_{k=0}^{\ell} a_{k} x_{1}^{\nu_{k, 1}} \cdots x_{n}^{v_{k, n}} .
$$


An immediate consequence of Definition 1.1 is the following useful property.

Remark A.1 If $S \subseteq \mathbb{R}^{n}$ is generic w.r.t. $\mathbb{V} \in \mathscr{V}_{n}^{\text {prop }}(\mathbb{R})$, then any encompassing set $\widetilde{S} \supseteq S$ is generic w.r.t. $\mathbb{V}$.

Definition 1.1 can be simplified using one instead of finitely many polynomials.

Lemma A.2 Any set $\mathbb{V} \subseteq \mathbb{R}^{n}$ is an algebraic variety if, and only if, there is a polynomial $p \in \mathbb{R}\left[x_{1}, \ldots, x_{n}\right]$ so that $\mathbb{V}=p^{-1}(\{0\})$.

Proof The proof follows from $\bigcap_{i=1}^{k} p_{i}^{-1}(\{0\})=\left(\sum_{i=1}^{k} p_{i}^{2}\right)^{-1}(\{0\})$.

We use the well-known result that proper algebraic varieties are Lebesgue null sets.

Proposition A.3 [5, p.240] Let $\mathbb{V} \subseteq \mathbb{R}^{n}$ be an algebraic variety. Then, $\mathbb{V}$ is proper if, and only if, $\mathbb{V}$ is a closed Lebesgue null set.

Corollary A.4 If $\mathbb{V}_{1}, \mathbb{V}_{2} \in \mathscr{V}_{n}^{\text {prop }}\left(\mathbb{R}^{n}\right)$, then $\mathbb{V}_{1} \cap \mathbb{V}_{2} \in \mathscr{V}_{n}^{\text {prop }}\left(\mathbb{R}^{n}\right)$ and $\mathbb{V}_{1} \cup \mathbb{V}_{2} \in$ $\mathscr{V}_{n}^{\text {prop }}\left(\mathbb{R}^{n}\right)$.

Proof The intersection and union of algebraic varieties are algebraic varieties; see [11, p.50]. Since the intersection and union of finitely many Lebesgue null sets are null sets, the claim follows from Proposition A.3.

By Definition 1.1 and Remark A.1, we have the following corollary.

Corollary A.5 For any $S_{1}, S_{2} \subseteq \mathbb{R}^{n}$, we have:

(i) If $S_{1}, S_{2}$ are generic sets, then $S_{1}^{c}$ is a Lebesgue null set and $S_{1} \cup S_{2}$ is a generic set.

(ii) $S_{1}$ and $S_{2}$ are generic sets if, and only if, $S_{1} \cap S_{2}$ is a generic set.

With the help of Corollary A.5, we conclude from Proposition A.3 that $\mathbb{R}^{n}$ cannot be partitioned into more than one generic set.

Lemma A.6 $\mathbb{R}^{n}$ cannot be partitioned into two generic sets.

Proof Seeking a contradiction, suppose $S_{1}, S_{2} \subseteq \mathbb{R}^{n}$ is a partition of $\mathbb{R}^{n}$ into generic sets, i.e., $S_{1}, S_{2}$ are nonempty disjoint sets with $S_{1} \cup S_{2}=\mathbb{R}^{n}$ and $S_{i}$ is generic w.r.t. $\mathbb{V}_{i} \in \mathscr{V}_{n}^{\text {prop }}(\mathbb{R})$, for $i=1,2$. Then, $S_{2}=S_{1}^{c} \subseteq \mathbb{V}_{1}$ and $S_{1}=S_{2}^{c} \subseteq \mathbb{V}_{2}$. This yields $\mathbb{V}_{1} \cup \mathbb{V}_{2}=\mathbb{R}^{n}$. The latter is a proper algebraic variety by Corollary A.4, but this contradicts Proposition A.3.

Another consequence of Proposition A.3 is the following lemma, which will be used for the simplification of some proofs.

Lemma A.7 If $\lambda^{n}(S)<\infty$ for some set $S \subseteq \mathbb{R}^{n}$, then $S$ is not generic.

Proof If $S \subseteq \mathbb{R}^{n}$ were a generic set with $\lambda^{n}(S)<\infty$, then $S^{c}$ is by Corollary A.5 a Lebesgue null set and additivity of the Lebesgue measure yields the contradiction

$$
\infty=\lambda^{n}\left(\mathbb{R}^{n}\right)=\lambda^{n}\left(S \cup S^{c}\right)=\lambda^{n}(S)+\lambda^{n}\left(S^{c}\right)=\lambda^{n}(S)<\infty .
$$


A useful property of generic sets is the next lemma.

Lemma A.8 Let $S_{1} \subseteq \mathbb{R}^{n}$ be generic and $S_{2}, S \subseteq \mathbb{R}^{n}$ with $S \cap S_{1}=S_{2} \cap S_{1}$. Then, $S$ is generic if, and only if, $S_{2}$ is generic.

Proof Since the statement is symmetric, only one direction has to be shown. Suppose $S$ is generic. Then, Corollary A.5 yields that $S \cap S_{1}=S_{2} \cap S_{1}$ is generic. Since $S_{2} \cap S_{1} \subseteq$ $S_{2}$, genericity of $S_{2}$ follows from Remark A.1.

\section{Appendix B Linear algebra}

We fix, throughout this section, the positive integers $\ell, n, m, d \in \mathbb{N}^{*}$.

Different controllability and stabilizability concepts can-as shown in Proposition 2.1 and 3.1 - be characterized by rank properties of certain matrices in terms of $(E, A, B) \in \Sigma_{\ell, n, m}$. We prove in the present section that these rank properties hold on generic subsets of $\Sigma_{\ell, n, m}$, where the latter matrix space is identified with $\mathbb{R}^{\ell(2 n+m)}$.

We recall the concept of a minor.

Definition B.1 (Submatrix, minor) Let $s, t, u, v \in \mathbb{N}^{*}$ fulfill the inequalities $s \leq u$ and $t \leq v$, let $\sigma: \underline{s} \rightarrow \underline{u}$ and $\pi: \underline{t} \rightarrow \underline{v}$ be injective, and let further $R=\mathbb{R}$ or $R=\mathbb{R}[x]$. Then, $\sigma$ and $\pi$ induce the submatrix

$$
m_{\sigma, \pi}: R^{u \times v} \rightarrow R^{s \times t}, \quad A \mapsto\left[A_{\sigma(i), \pi(j)}\right]_{i \in \underline{s}, j \in \underline{t}} .
$$

If $s=t=d$, then the mapping

$$
M_{\sigma, \pi}: R^{u \times v} \rightarrow R, \quad A \mapsto \operatorname{det} m_{\sigma, \pi}(A)
$$

is called minor of degree $d$ (w.r.t. $R^{u \times v}$ ).

$\diamond$

Remark B.2 Let $M_{\sigma, \pi}$ be a minor of order $d$ w.r.t. $\mathbb{R}^{u \times v}$ and $S_{d}$ the set of all permutations of a $d$-element family. Then, the Leibniz formula gives

$$
\forall A \in \mathbb{R}^{u \times v}: \quad M_{\sigma, \pi}(A)=\sum_{\tau \in S_{d}} \operatorname{sign} \tau \prod_{i=1}^{d} A_{\sigma(i), \pi(\tau(i))},
$$

and hence, $M_{\sigma, \pi}$ is a polynomial in the entries of the matrix and we may write

$$
M_{\sigma, \pi} \in \mathbb{R}\left[x_{1}, \ldots, x_{u v}\right]
$$

Next, we generalize the well-known property that invertibility of squared real matrices is a generic property to block matrices. 
Proposition B.3 The following sets are proper algebraic varieties

(i) $\left\{(E, A, B) \in \Sigma_{\ell, n, m} \mid \operatorname{rk}[E, A, B]<d\right\}$ if, and only if, $d \leq \min \{\ell, 2 n+m\}$;

(ii) $\quad\left\{(E, A, B) \in \Sigma_{\ell, n, m} \mid \operatorname{rk}[E, B]<d\right\}$ if, and only if, $d \leq \min \{\ell, n+m\}$;

(iii) $\quad\left\{(E, A, B) \in \Sigma_{\ell, n, m} \mid \operatorname{rk} E<d\right\}$ if, and only if, $d \leq \min \{\ell, n\}$;

Proof (i) Set $S^{c}:=\left\{(E, A, B) \in \Sigma_{\ell, n, m} \mid \operatorname{rk}[E, A, B]<d\right\}$.

$\Longrightarrow$ If $d>\min \{\ell, 2 n+m\}$, then $S^{c}=\Sigma_{\ell, n, m}$ is an algebraic variety but not proper.

$\Leftarrow$ Let $\tilde{M}_{1}(\cdot), \ldots, \tilde{M}_{r}(\cdot)$ be all minors of order $d$ w.r.t. $\mathbb{R}^{\ell \times(2 n+m)}$ and define

$$
\forall i \in \underline{r}: \quad M_{i}: \Sigma_{\ell, n, m} \rightarrow \mathbb{R}, \quad(E, A, B) \mapsto \tilde{M}_{i}([E, A, B]) .
$$

The fact (see the German reference [6, Sect.3.3.6], for an English reference we only know [2, Th.4.6.1] who prove necessity, only)

$$
\operatorname{rk}[E, A, B]<d \quad \Longleftrightarrow \quad \forall i \in \underline{r}: M_{i}(E, A, B)=0
$$

yields $S^{c}=\bigcap_{i=1}^{r}\left(M_{i}\right)^{-1}(\{0\})$. Now, Remark B.2 yields that every $\left(M_{i}\right)^{-1}(\{0\})$ is an algebraic variety, whence $S^{c}$ an algebraic variety by Corollary A.5 (ii). Since $S$ is nonempty, $S^{c}$ is a proper algebraic variety.

The remaining proofs of (ii)-(iv) are similar and omitted.

In the remainder of this section, we investigate polynomial matrices of degree one, also called a pencil, of the form

$$
[s E-A, B] \in \mathbb{R}[s]^{\ell \times(n+m)}, \quad \text { where }(E, A, B) \in \Sigma_{\ell, n, m} .
$$

Remark B.4 Let $\tilde{M}$ be any minor of order $d \leq \min \{\ell, n+m\}$ w.r.t. $\mathbb{R}[s]^{\ell \times(n+m)}$. Then, $\widetilde{M}$ induces the mapping

$$
M: \Sigma_{\ell, n, m} \rightarrow \mathbb{R}[s], \quad(E, A, B) \mapsto \tilde{M}([s E-A, B])
$$

and Leibniz' formula yields the existence of $p_{0}, \ldots, p_{d} \in \mathbb{R}\left[s_{1}, \ldots, s_{\ell(2 n+m)}\right]$ so that

$$
\forall(E, A, B) \in \Sigma_{\ell, n, m}: \quad M(E, A, B)=\sum_{i=0}^{d} p_{i}(E, A, B) s^{i}
$$

In passing note that $p_{0}(E, A, B)=\tilde{M}([-A, B])$.

The Eq. (7) sets us in a position to characterize when the set of matrix triples $(E, A, B)$ with pencil $[s E-A, B]$ of "full" rank w.r.t. the field of rational functions is generic. 
Proposition B.5 The set

$$
S^{c}=\left\{(E, A, B) \in \Sigma_{\ell, n, m} \mid \operatorname{rk}_{\mathbb{R}(s)}[s E-A, B]<d\right\}
$$

is a proper algebraic variety if, and only if, $d \leq \min \{\ell, n+m\}$.

Proof $\Longrightarrow$ If $S^{c}$ is a proper algebraic variety, then $S \neq \emptyset$. The latter holds if, and only if, there exists some $(E, A, B) \in \Sigma_{\ell, n, m}$ so that $d \leq \operatorname{rk}_{\mathbb{R}(s)}[s E-A, B] \leq$ $\min \{\ell, n+m\}$.

$\Leftarrow$ Let $\widetilde{M}_{1}, \ldots, \widetilde{M}_{r}$ be all minors of order $d$ w.r.t. $\mathbb{R}[s]^{\ell \times(n+m)}$ and $M_{i}$ as in (7) the mapping $M_{i}$ induced by $\widetilde{M}_{i}$ on $\Sigma_{\ell, n, m}$ as in (7) on $\Sigma_{\ell, n, m}$.

Note that $r \geq 1$ since $d \leq \min \{\ell, n+m\}$. Then,

$\operatorname{rk}_{\mathbb{R}(s)}[s E-A, B]<d \quad \Longleftrightarrow \quad \forall i \in \underline{r}: M_{i}(E, A, B)=\tilde{M}_{i}([s E-A, B])=0$.

Introducing the mapping

$$
\varphi:\{p \in \mathbb{R}[s]: \operatorname{deg} p \leq d\} \rightarrow \mathbb{R}^{d+1}, \quad p=\sum_{j=0}^{d} p_{j} s^{j} \mapsto\left(p_{0}, \ldots, p_{d}\right)
$$

we see that the functions $h_{i}:=\varphi \circ M_{i}: \Sigma_{\ell, n, m} \rightarrow \mathbb{R}^{d+1}$, defined for all $i \in \underline{r}$, are well defined polynomial vectors and satisfy, in view of (8), the equivalence

$$
\operatorname{rk}_{\mathbb{R}(s)}[s E-A, B]<d \quad \Longleftrightarrow \quad \forall i \in \underline{r}: h_{i}(E, A, B)=0
$$

Therefore, $S^{c}=\bigcap_{i=1}^{r} h_{i}^{-1}(\{0\})$. Now, Remark B.2 yields that every $\left(M_{i}\right)^{-1}(\{0\})$ is an algebraic variety, whence $S^{c}$ an algebraic variety by Corollary A.5 (ii).

It remains to show that $S^{c}$ is proper. Since each $h_{i}$ is not identical zero,$h_{i}^{-1}(\{0\})$ is a proper algebraic variety, and so is $S^{c}$ by Corollary A.4. This completes the proof.

We will now study matrix triples $(E, A, B) \in \Sigma_{\ell, n, m}$ so that the polynomial matrix $[s E-A, B]$ has "full" rank for each $s=\lambda \in \mathbb{C}$. An important tool for these investigations is coprime polynomials and the characterization by the resultant. 
Definition B.6 (Resultant, [7, p.61]) The resultant of two polynomials $p(s), q(s) \in$ $\mathbb{R}[s] \backslash\{0 \mathbb{R}[s]\}$ with $\operatorname{deg} p=n \geq 0$ and $\operatorname{deg} q=m \geq 0$ is defined as

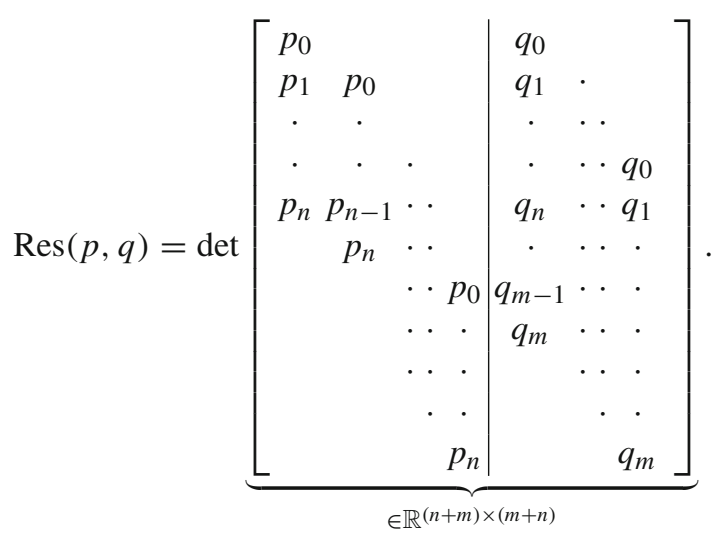

The matrix above is called the Sylvester matrix of $p(s)$ and $q(s)$. The Sylvester matrix contains $m$ columns with the coefficients of $p$ and $n$ columns with the coefficients of $q$, so that it is in $\mathbb{R}^{(n+m) \times(m+n)}$. All other entries are zero. Note that the diagram shows the case $n<m$.

A well-known characterization of coprime polynomials in terms of the Sylvester matrix is given in the following lemma; for a proof see for example [7, Thm. 3.3.1].

Lemma B.7 Let $p(s), q(s) \in \mathbb{R}[s] \backslash\left\{0_{\mathbb{R}[s]}\right\} \subseteq \mathbb{C}[s] \backslash\left\{0_{\mathbb{C}[s]}\right\}$. Then, $\operatorname{Res}(p, q)$ is welldefined and $p(s)$ and $q(s)$ are not coprime (i.e., there is some common zero $z \in \mathbb{C}$ such that $p(z)=q(z)=0)$ if, and only if, $\operatorname{Res}(p, q)=0$.

We are now in a position the characterize genericity of a set of pencils satisfying a rank condition in the complex plane.

Proposition B.8 The set

$$
S=\left\{(E, A, B) \in \Sigma_{\ell, n, m} \mid \forall \lambda \in \mathbb{C}: \operatorname{rk} \mathbb{C}[\lambda E-A, B] \geq d\right\}
$$

is generic if, and only if,

$$
d \leq \min \{\ell, n+m\} \quad \wedge \neg(d=\ell=n+m) .
$$

Proof $\Longrightarrow \quad$ Assume first $d>\min \{\ell, n+m\}$. Then, $S=\emptyset$ and hence $S$ is not generic.

Assume next $d=\ell=n+m$.

The set

$$
\widetilde{S}^{c}:=\left\{(E, A, B) \in \Sigma_{\ell, n, m} \mid \operatorname{det}[E, B]=0\right\}
$$


is a proper algebraic variety by Proposition B.3(ii). Thus, $\widetilde{S}$ is a generic set. Since

$$
\widetilde{S} \subseteq S^{\prime}:=\left\{(E, A, B) \in \Sigma_{\ell, n, m} \mid \operatorname{deg} \operatorname{det}[s E-A, B] \geq 1\right\},
$$

Remark A.1 gives that $S^{\prime}$ is a generic set. The fundamental theorem of algebra implies $S^{\prime} \subseteq S^{c}$, and a repeated application of Remark A.1 gives that $S^{c}$ is a generic set, too. Hence, Lemma A.6 implies that $S$ is not generic.

$\Leftarrow$ We proceed in step $\underset{\sim}{\sim}$.

STEP 1 Let $\widetilde{M}_{1}, \ldots, \widetilde{M}_{r}$ be all minors of order $d \leq \min \{\ell, n+m\}$ w.r.t. $\mathbb{R}[s]^{\ell \times(n+m)}$ so that $\tilde{M}_{i} \neq \widetilde{M}_{j}$ for all $i \neq j \in \underline{r}$ and define the induced mappings on $\Sigma_{\ell, n, m}$ by

$$
\forall i \in \underline{r}: M_{i}: \Sigma_{\ell, n, m} \rightarrow \mathbb{R}[s], \quad(E, A, B) \mapsto \tilde{M}_{i}([s E-A, B]) .
$$

Since $\neg(d=\ell=n+m)$ and $d \leq \min \{\ell, n+m\}$, we conclude $d<\max \{\ell, n+m\}$, and therefore $r \geq 2$. Now, we are in a position to show the following implications for any $(E, A, B) \in \Sigma_{\ell, n, m}$ and any $\lambda \in \mathbb{C}$ :

$$
\begin{aligned}
& \exists i, j \in \underline{r}: M_{i}(E, A, B) \text { and } M_{j}(E, A, B) \text { are coprime } \\
\Longrightarrow & \exists i, j \in \underline{r}: \lambda \text { is not a common zero of } M_{i}(E, A, B), M_{j}(E, A, B) \\
\Longleftrightarrow \quad & \exists i \in \underline{r}: M_{i}(E, A, B)(\lambda) \neq 0 \\
\Longleftrightarrow & \operatorname{rk} \mathbb{C}[\lambda E-A, B] \geq d .
\end{aligned}
$$

Therefore,

$$
\widetilde{S}:=\left\{(E, A, B) \in \Sigma_{\ell, n, m} \mid(9) \text { holds }\right\} \subseteq S
$$

STEP 2 Define $N:=\ell(2 n+m)$ and, for any $i \in \underline{r}$, the maximal degree of the image of $\Sigma_{\ell, n, m}$ under $M_{i}$ as

$$
\alpha_{i}:=\max \left\{\operatorname{deg} M_{i}(E, A, B) \mid(E, A, B) \in \Sigma_{\ell, n, m}\right\}
$$

and note that not all $\alpha_{i}$ 's are zero. Remark B.4 allows to write the minors w.r.t. the polynomial ring $\mathbb{R}[s]$ as

$$
\begin{aligned}
& \forall i \in \underline{r} \quad \exists M_{i}^{0}, \ldots, M_{i}^{\alpha_{i}} \in \mathbb{R}\left[s_{1}, \ldots, s_{N}\right] \forall(E, A, B) \in \Sigma_{\ell, n, m}: \\
& M_{i}(E, A, B)=\sum_{j=0}^{\alpha_{i}} M_{i}^{j}(E, A, B) s^{j}
\end{aligned}
$$

and, by the definition of $\alpha_{i}$, we find that $M_{i}^{\alpha_{i}} \neq 0$ for all $i \in \underline{r}$. Choose $k \in \underline{r}$ so that

$$
\alpha_{k}=\max \left\{\alpha_{i} \mid i \in \underline{r}\right\}>0 .
$$

STEP 3 Setting

$$
\widehat{S}:=\left(M_{k}^{\alpha_{k}}\right)^{-1}(\{0\})^{c},
$$


we show that $(\widehat{S} \cap \widetilde{S})^{c} \subseteq \mathbb{V}$ for some $\mathbb{V} \in \mathscr{V}_{N}^{\text {prop }}(\mathbb{R})$.

STEP 3A We first show that $(\widehat{S} \cap \widetilde{S})^{c} \subseteq \mathbb{V}$ for some $\mathbb{V} \in \mathscr{V}_{N}(\mathbb{R})$. Define,

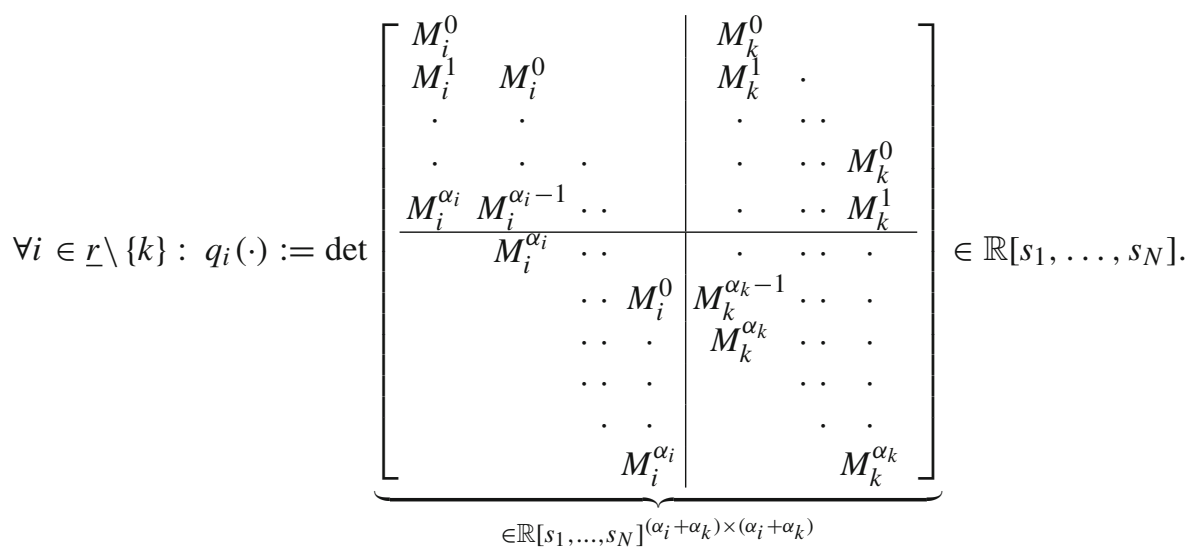

Laplace's formula and expanding the last row yields

$\forall i \in \underline{r} \backslash\{k\} \forall(E, A, B) \in \widehat{S}$ with $M_{i}(E, A, B) \neq 0$ :

$q_{i}(E, A, B)= \pm\left(M_{k}^{\alpha_{k}}(E, A, B)\right)^{\alpha_{i}-\operatorname{deg} M_{i}(E, A, B)} \operatorname{Res}\left(M_{i}(E, A, B), M_{k}(E, A, B)\right)$.

Recall that on $\widehat{S}$ we have $M_{k}^{\alpha_{k}}(E, A, B) \neq 0$, and hence in particular $M_{k}(E, A, B) \neq$ 0 . Thus, (12) in conjunction with Lemma B.7 gives

$$
\begin{aligned}
& \forall(E, A, B) \in \widehat{S} \text { with } M_{i}(E, A, B) \neq 0 \forall i \in \underline{r} \backslash\{k\}: \\
& q_{i}(E, A, B)=0 \quad \Longleftrightarrow \quad M_{i}(E, A, B), M_{k}(E, A, B) \text { are not coprime. (13) }
\end{aligned}
$$

If, on the other hand, $M_{i}(E, A, B)=0$ for some $(E, A, B) \in \widehat{S}$, then the matrix in the definition of $q_{i}$ contains at least one zero collumn; hence, its determinant is zero and thus $q_{i}(E, A, B)=0$. Since $\operatorname{deg} M_{k}(E, A, B)=\alpha_{k}>0, M_{k}(E, A, B)$ has at least one zero and $M_{i}(E, A, B)$ and $M_{k}(E, A, B)$ are not coprime. This proves that (13) applies to all $(E, A, B) \in \widehat{S}$. Finally, we arrive at the inclusion

$$
(\widehat{S} \cap \widetilde{S})^{c}=(\widehat{S})^{c} \cup(\widetilde{S})^{c} \subseteq \mathbb{V}:=\left(M_{k}^{\alpha_{k}}\right)^{-1}(\{0\}) \cup \bigcap_{i=1, i \neq k}^{r} q_{i}^{-1}(\{0\}) \in \mathscr{V}_{N}(\mathbb{R})
$$

STEP 3B It remains to show that $\mathbb{V}$ is proper. Equivalently, we prove that there is a $(E, A, B) \in \Sigma_{\ell, n, m}$ so that $\operatorname{deg} M_{k}(E, A, B)=\alpha_{k}$ and $M_{k}(E, A, B)$ and $M_{i}(E, A, B)$ are coprime for some $i \in \underline{r} \backslash\{k\}$. Let

$$
a_{i, j} \in \mathbb{R} \text { for }(i, j) \in\{(i, j) \in \underline{\ell} \times \underline{n} \mid j=i \vee j=i+1 \vee j+1=i\}
$$


be pairwise different real numbers.

Consider the case $\ell<n+m$. Define $E, A \in \mathbb{R}^{\ell \times n}$ by

$$
\forall(i, j) \in \underline{\ell} \times \underline{n}: E_{i, j}:=\left\{\begin{array}{ll}
1, & j \in\{i, i+1\} \\
0, & \text { else }
\end{array}, \quad A_{i, j}:= \begin{cases}a_{i, j}, & j \in\{i, i+1\} \\
0, & \text { else }\end{cases}\right.
$$

and $B \in \mathbb{R}^{\ell \times m}$ by

$$
\forall(i, j) \in \underline{\ell} \times \underline{m}: B_{i, j}:= \begin{cases}1, & i \geq n+1, j \in i-n, i-n+1 \\ 0, & \text { else. }\end{cases}
$$

Then, $(E, A, B) \in \Sigma_{\ell, n, m}$ and the pencil $[s E-A, B]$ has the structure

$$
[s E-A, B]=\left[\begin{array}{ccccccccc}
\star & \star & 0 & \cdots & 0 & 0 & 0 & \cdots & 0 \\
0 & \star & \star & \cdots & 0 & 0 & 0 & \cdots & 0 \\
0 & 0 & \star & \cdots & 0 & 0 & 0 & \cdots & 0 \\
\vdots & \vdots & \ddots & \ddots & \vdots & \vdots & \vdots & \ddots & \vdots \\
0 & 0 & 0 & \cdots & \star & \star & 0 & \cdots & 0 \\
0 & 0 & 0 & \cdots & 0 & \star & \star & \cdots & 0
\end{array}\right]
$$

W.1.o.g. let $k=1$ and

$$
\begin{aligned}
& M_{1}: \Sigma_{\ell, n, m} \rightarrow \mathbb{R}[x], \quad(E, A, B) \mapsto \operatorname{det}([s E-A, B])_{i, j \in \underline{d}}, \\
& M_{2}: \Sigma_{\ell, n, m} \rightarrow \mathbb{R}[x], \quad(E, A, B) \mapsto \operatorname{det}([s E-A, B])_{i, j-1 \in \underline{d}} .
\end{aligned}
$$

In passing note that $(E, A, B) \in \Sigma_{\ell, n, m}$ constructed above fulfills

$$
M_{1}(E, A, B)=\prod_{i=1}^{\min \{d, n\}}\left(s-a_{i, i}\right) \text { and } M_{2}(E, A, B)=\prod_{i=1}^{\min \{d, n\}}\left(s-a_{i, i+1}\right),
$$

$M_{1}(E, A, B), M_{2}(E, A, B)$ are coprime by our choice of $a_{i, j}$, and they have maximal degree.

If $\ell \geq n+m$, then a similar argument, i.e., taking the determinant of the first two upper right $d \times d$ submatrices of the pencil, proves the claim.

STEP 4 Finally, $S^{c} \stackrel{(10)}{\subseteq}(\widehat{S} \cap \widetilde{S})^{c}$ in conjunction with (14) yields genericity of the set $S$.

This completes the proof of the proposition.

If we consider stabilizability of systems described by differential-algebraic equation, then we are interested in the rank of the matrix $[\lambda E-A, B]$ for $\lambda$ belonging to the closed right half plane only. 
Proposition B.9 The set

$$
S=\left\{(E, A, B) \in \Sigma_{\ell, n, m} \mid \forall \lambda \in \overline{\mathbb{C}}_{+}: \operatorname{rk} \mathbb{C}[\lambda E-A, B] \geq d\right\}
$$

is generic if, and only if,

$$
d \leq \min \{\ell, n+m\} \wedge \neg(d=\ell=n+m) .
$$

Proof $\Longrightarrow \quad$ Note that $S \neq \varnothing$ if, and only if, $d \leq \min \{\ell, n+m\}$. Assume $d=$ $\ell=n+m$. By the well-known Hurwitz criterion (see [8, pp.339]), we find that a polynomial whose coefficients do not have the same sign has at least one zero in the closed right half plane. Consider the set of $(E, A, B) \in \Sigma_{(n+m), n, m}$ so that the coefficients of $\operatorname{det}[s E-A, B]$ do not have the same sign. Then, this set is nonempty, open and is included in $S^{c}$. Openness yields that $S^{c}$ has a positive Lebesgue measure and thus $S$ is not generic Corollary A.5(i).

$\Leftarrow$ This is a consequence of Proposition B.8.

\section{References}

1. Banaszuk A, Przyłuski KM (1999) On perturbations of controllable implicit linear systems. IMA J Math Control Inf 16:91-102

2. Barker GP, Schneider H (1973) Matrices and linear algebra, 2nd edn. Dover Publications, New York

3. Belur MN, Shankar S (2019) The persistence of impulse controllability. Math Control Signals Syst 31:487-501

4. Berger T, Reis T (2013) Controllability of linear differential-algebraic systems - a survey. In: Ilchmann A, Reis T (eds) Surveys in differential-algebraic equations I, Differential-Algebraic Equations Forum. Springer, Berlin, pp 1-61. https://doi.org/10.1007/978-3-642-34928-7_1

5. Federer H (1969) Geometric measure theory. Springer, Berlin

6. Fischer G (2014) Linear algebra, 18th edn. Springer Spektrum, Wiesbaden

7. Fuhrmann PA (2012) A polynomial approach to linear algebra, 2nd edn. Springer, New York

8. Hinrichsen D, Pritchard AJ (2005) Mathematical systems theory I. Modelling, state space analysis, stability and robustness. Springer, Berlin

9. Kirchhoff J (2021) Linear port-Hamiltonian systems are generically controllable. Conditionally accepted IEEE-TAC, preprint available at arXiv:2104:02111

10. Lee EB, Markus L (1967) Foundations of optimal control theory. Wiley, New York

11. Reid M (1988) Undergraduate algebraic geometry. Cambridge University Press, Cambridge

12. Wonham WM (1974) Linear multivariable control: a geometric approach, 1st edn. Springer, Heidelberg

13. Wonham WM (1985) Linear multivariable control: a geometric approach, 3rd edn. Springer, New York

Publisher's Note Springer Nature remains neutral with regard to jurisdictional claims in published maps and institutional affiliations. 